\title{
Factors that contribute to long-term survival in patients with leukemia not in remission at allogeneic hematopoietic cell transplantation
}

Hideo Koh', Hirohisa Nakamae ${ }^{1 *}$, Kiyoyuki Hagihara ${ }^{1}$, Takahiko Nakane1, Masahiro Manabe', Yoshiki Hayashi ${ }^{1}$, Mitsutaka Nishimoto', Yukari Umemoto', Mika Nakamae', Asao Hirose', Eri Inoue ${ }^{1}$, Atsushi Inoue1, Masahiro Yoshida', Masato Bingo ${ }^{1}$, Hiroshi Okamura', Ran Aimoto ${ }^{1}$, Mizuki Aimoto ${ }^{1}$, Yoshiki Terada', Ki-Ryang Koh', Takahisa Yamane', Masahiko Ohsawa² and Masayuki Hino'

\begin{abstract}
Background: There has been insufficient examination of the factors affecting long-term survival of more than 5 years in patients with leukemia that is not in remission at transplantation.

Method: We retrospectively analyzed leukemia not in remission at allogeneic hematopoietic cell transplantation (allo-HCT) performed at our institution between January 1999 and July 2009. Forty-two patients with a median age of 39 years received intensified conditioning $(n=9)$, standard $(n=12)$ or reduced-intensity conditioning $(n=21)$ for allo-HCT. Fourteen patients received individual chemotherapy for cytoreduction during the three weeks prior to reduced-intensity conditioning. Diagnoses comprised acute leukemia $(n=29)$, chronic myeloid leukemia-accelerated phase $(n=2)$, myelodysplastic syndrome/acute myeloid leukemia (MDS/AML) $(n=10)$ and plasma cell leukemia $(n=1)$. In those with acute leukemia, cytogenetic abnormalities were intermediate $(44 \%)$ or poor (56\%). The median number of blast cells in bone marrow (BM) was 26.0\% (range; 0.2-100) before the start of chemotherapy for allo-HCT. Six patients had leukemic involvement of the central nervous system. Stem cell sources were related BM (7\%), related peripheral blood (31\%), unrelated BM (48\%) and unrelated cord blood (CB) (14\%).
\end{abstract}

Results: Engraftment was achieved in 33 (79\%) of 42 patients. Median time to engraftment was 17 days (range: 9-32). At five years, the cumulative probabilities of acute graft-versus-host disease (GVHD) and chronic GVHD were $63 \%$ and $37 \%$, respectively. With a median follow-up of 85 months for surviving patients, the five-year Kaplan-Meier estimates of leukemia-free survival rate and overall survival (OS) were $17 \%$ and $19 \%$, respectively. At five years, the cumulative probability of non-relapse mortality was $38 \%$. In the univariable analyses of the influence of pre-transplant variables on OS, poor-risk cytogenetics, number of BM blasts (>26\%), MDS overt AML and $C B$ as stem cell source were significantly associated with worse prognosis $(p=.03, p=.01, p=.02$ and $p<.001$, respectively). In addition, based on a landmark analysis at 6 months post-transplant, the five-year Kaplan-Meier estimates of OS in patients with and without prior history of chronic GVHD were 64\% and 17\% $(p=.022)$, respectively.

Conclusion: Graft-versus-leukemia effects possibly mediated by chronic GVHD may have played a crucial role in long-term survival in, or cure of active leukemia.

\footnotetext{
* Correspondence: hirohisa@msic.med.osaka-cu.ac.jp

${ }^{1}$ Hematology, Graduate School of Medicine, Osaka City University, Osaka,

Japan

Full list of author information is available at the end of the article
} 


\section{Introduction}

Patients with primary refractory or refractory relapsed acute leukemia have an extremely poor prognosis. It has been generally recognized that few cases with primary refractory or refractory relapsed acute leukemia can be cured using conventional chemotherapy alone [1]. While allogeneic hematopoietic cell transplantation (allo-HCT) has the potential to cure even active leukemia, it has not been determined what subgroup can receive a long-term benefit from it.

Several retrospective studies have reported the prognostic factors for allo-HCT in patients not in remission at allo-HCT including untreated first relapse cases [2-8]. However, the factors contributing to long-term survival have not been established because the follow-up periods of these studies were not long enough at less than five years. Importantly, it can be assumed that patients who survive for more than five years without leukemia relapse are most likely cured. Only one large-scale retrospective study has examined long-term outcomes for more than five years following allo-HCT in adult patients with acute leukemia not in remission [9]. This study showed that several pre-transplant variables including complete remission duration, type of donor, disease burden, performance status, age and cytogenetics affected survival. However, whether post-transplant variables such as acute or chronic graft-versus-host disease (GVHD) influenced the post-HCT prognosis was not assessed. To our knowledge, no studies have investigated pre- and/or post-transplant factors which are associated with long-term survival exclusively in adult patients with active leukemia at allo-HCT. Therefore, we comprehensively evaluated the pre- and post-transplant factors which contribute to long-term survival of more than five years in patients with leukemia not in remission at allo-HCT.

\section{Patients and methods}

Between January 1999 and July 2009, 42 consecutive patients (24 males and 18 females) with leukemia not in remission, aged 15 to 67 years (median age: 39 years), underwent allo-HCT at our institution. Patients with de novo acute myeloid leukemia (AML; $\mathrm{n}=17$ ), acute lymphoblastic leukemia (ALL; $\mathrm{n}=12$ ), chronic myeloid leukemia in accelerated phase (CML-AP; $\mathrm{n}=2$ ), myelodysplastic syndrome (MDS) overt AML $(\mathrm{n}=10)$ and plasma cell leukemia $(n=1)$ were included. Highrisk AML was defined according to the Eastern Cooperative Oncology Group/Southwest Oncology Group classification as having poor-risk cytogenetics (5/del[5q], 7/del[7q], inv[3q], abn11q, 20q or 21q, del[9q], t[6;9], t [9;22], abn17p, and complex karyotype defined as three or more abnormalities) [10]. High-risk ALL was defined as having poor-risk cytogenetics with either $\mathrm{t}(4: 11), \mathrm{t}$ $(9 ; 22), t(8 ; 14)$, hypodiploidy or near triploidy, or more than five cytogenetic abnormalities [11]. Of study subjects with acute leukemia, cytogenetic abnormalities were intermediate $(\mathrm{n}=17,44 \%)$ or poor $(\mathrm{n}=22,56 \%)$. Seven patients were primary refractory to induction chemotherapy. The other patients relapsed after conventional chemotherapy $(n=23)$ or the first or the second HCT $(n=9)$. The median number of blast cells in bone marrow (BM) was $26.0 \%$ (range; $0.2-100$ ) before the start of chemotherapy for allo-HCT. Six patients had leukemic involvement of the central nervous system (CNS). Stem cell sources were related BM $(n=3,7 \%)$, related peripheral blood (PB) $(\mathrm{n}=13,31 \%)$, unrelated $\mathrm{BM}(\mathrm{n}=20,48 \%)$ and unrelated cord blood $(\mathrm{CB})(\mathrm{n}=$ 6, 14\%). Standard serologic typing was used for human leukocyte antigen (HLA) -A, B and DRB1. Thirty-one pairs were matched for HLA-A, B and DRB1 antigens. Three patients were mismatched for one HLA antigen (two at HLA-A, one at HLA-B), and seven were mismatched for two (two at HLA-A and B, five (all CB) at HLA-B and DRB1). The remaining one patient was mismatched for all three antigens (haploidentical). We classified conditioning regimens into four categories. Standard conditioning $(\mathrm{n}=12)$ comprised a busulfanbased or total body irradiation (TBI)-based (12Gy) regimen. Busulfan was given as a total of $16 \mathrm{mg} / \mathrm{kg}$ orally or equivalent dose, $12.8 \mathrm{mg} / \mathrm{kg}$ intravenously (i.v.). Intensified conditioning $(\mathrm{n}=9)$ consisted of additional cytoreductive chemotherapy in the three weeks before conditioning, followed by standard conditioning. Of the 21 patients receiving standard or intensified conditioning, 13 patients received the TBI-based regimen. Reduced-intensity conditioning $(\mathrm{n}=21)$ comprised a fludarabine-based $(\mathrm{n}=20)$ and cladribine-based regimen $(\mathrm{n}=1)$. Fludarabine was given as $25-35 \mathrm{mg} / \mathrm{m}^{2}$ i.v. on five or six consecutive days. Of the 21 patients receiving reduced-intensity conditioning, 14 patients received cytoreductive chemotherapy in the three weeks before conditioning. Prophylaxis for acute GVHD was a calcineurin inhibitor alone $(\mathrm{n}=5)$, calcineurin inhibitor plus short-term methotrexate $(\mathrm{n}=32)$, calcineurin inhibitor plus mycophenolate mofetil $(n=2)$, or none $(n=3)$. The calcineurin inhibitor included cyclosporine administered to 33 patients and tacrolimus to six patients.

\section{End points}

The absence of post-transplant remission in some patients biased the calculation of relapse rate, nonrelapse mortality (NRM) and leukemia-free survival (LFS). Therefore, we set five-year overall survival (OS) as the primary end point. OS was defined as time from the date of last transplantation to the date of death or 
last follow-up. LFS was defined as time from the date of last transplantation to the date of disease relapse, death during remission or last follow-up. NRM was defined as a death not related to disease. Neutrophil recovery was defined as an absolute neutrophil count of at least 500 cells $/ \mathrm{mm}^{3}$ for three consecutive time points. Platelet recovery was defined as a count of at least 20000 platelets $/ \mathrm{mm}^{3}$ without transfusion support. Acute GVHD (aGVHD) was defined in accordance with standard criteria [12]. Chronic GVHD (cGVHD) was evaluated in patients surviving for more than 100 days after allo-HCT and was classified into limited or extensive type [13].

\section{Statistical analysis}

If the disease for which the patient underwent transplantation was present at the time of death or found at autopsy, we defined disease relapse/progression as the primary cause of death. Unadjusted survival probabilities were estimated using the Kaplan and Meier method and compared using the log-rank tests. Cumulative incidence curves were used in a competing-risks model to calculate the probability of aGVHD, cGVHD and NRM [14]. For neutrophil and platelet recovery, death before neutrophil or platelet recovery was the competing event; for GVHD, death without GVHD and relapse were the competing events; and, for NRM, relapse was the competing event. In order to examine the impact of cGVHD on survival, we performed a landmark analysis, which divided patients according to their prior history of cGVHD at 6 months post-transplant [15]. We excluded from landmark analysis patients who died or relapsed less than 6 months after transplant, and did not use the information on whether or not patients developed cGVHD 6 months after transplant. Multivariable analysis of prognostic factors for the primary outcome could not be conducted due to lack of statistical power. Instead, we performed a landmark analysis, which divided patients according to the significant pre-transplant factors and their prior history of cGVHD at 6 months post-transplant. All $P$ values were 2 -tailed and considered statistically significant if the values were less than 0.05. All statistical analyses were performed using the PASW Statistics17.0 (SPSS Inc, Chicago, IL, USA) and the statistical software environment $\mathrm{R}$, version 2.9.1.

\section{Results}

The baseline characteristics of the patients are shown in Table 1.

\section{Engraftment}

Neutrophil engraftment was achieved in 33 (79\%) of 42 patients. The median time to neutrophil engraftment was 17 days (range, 9-32). In a total of four of 27 evaluable
Table 1 Baseline characteristics of study participants

\begin{tabular}{|c|c|c|}
\hline Variable & n (\%) & $\begin{array}{l}\text { Median } \\
\text { (Range) }\end{array}$ \\
\hline Male sex & $24(57.1)$ & \\
\hline \multicolumn{3}{|l|}{ Diagnosis } \\
\hline de novo AML & $17(40.5)$ & \\
\hline ALL & $12(28.6)$ & \\
\hline CML-AP & $2(4.8)$ & \\
\hline MDS overt AML & $10(23.8)$ & \\
\hline $\mathrm{PCL}$ & $1(2.4)$ & \\
\hline \multicolumn{3}{|l|}{ Cytogenetics } \\
\hline Intermediate & 17 & \\
\hline Poor & 22 & \\
\hline \multicolumn{3}{|l|}{ ECOG PS } \\
\hline 0 & $2(4.8)$ & \\
\hline 1 & $25(59.5)$ & \\
\hline 2 & $7(16.7)$ & \\
\hline 3 & $8(19.0)$ & \\
\hline \multicolumn{3}{|l|}{ Status at allo-HCT } \\
\hline $\begin{array}{l}\text { Primary refractory/Refractory } \\
\text { relapse/Untreated MDS overt AML }\end{array}$ & $7 / 32 / 3$ & \\
\hline No. chemo regimens prior allo-HCT & & $6(0-18)$ \\
\hline Time from diagnosis to allo-HCT (days) & & $319(23-3738)$ \\
\hline Marrow blasts at allo-HCT & & $26.0(0.2-100)$ \\
\hline \multicolumn{3}{|l|}{ Conditioning regimen } \\
\hline Intensified & $9(21.4)$ & \\
\hline Standard & $12(28.6)$ & \\
\hline Reduced-intensity & $7(16.7)$ & \\
\hline $\begin{array}{l}\text { Reduced-intensity + cytoreductive } \\
\text { chemotherapy }\end{array}$ & $14(33.3)$ & \\
\hline \multicolumn{3}{|l|}{ GVHD prophylaxis } \\
\hline None & $3(7.1)$ & \\
\hline Calcineurin inhibitor alone & $5(11.9)$ & \\
\hline Calcineurin inhibitor + sMTX & $32(76.2)$ & \\
\hline Calcineurin inhibitor + MMF & $2(4.8)$ & \\
\hline \multicolumn{3}{|l|}{ Donor (HLA-A, B and DRB1 antigens) } \\
\hline Matched related PB/BM & $10 / 2$ & \\
\hline Mismatched related PB/BM & $3 / 1$ & \\
\hline Matched unrelated BM & 19 & \\
\hline Mismatched unrelated BM & 1 & \\
\hline Umbilical cord blood & 6 & \\
\hline
\end{tabular}

allo-HCT: allogeneic hematopoietic cell transplantation; HLA: human leukocyte antigen; sMTX: short-term methotrexate; MMF: mycophenolate motefil; BM: bone marrow; PB: peripheral blood.

patients, a platelet count $>20000 / \mu \mathrm{l}$ was not achieved. In the patients that achieved platelet counts of $\geq 20000 /$ $\mu \mathrm{l}$, the median time to platelet engraftment was 33 days (range, 13-99). The cumulative probabilities of neutrophil and platelet engraftment were $79 \%$ and $55 \%$, respectively.

\section{GVHD}

Twenty-four of 42 patients developed aGVHD (eight grade I, nine grade II, five grade III, two grade IV). Twelve of 24 evaluable patients developed cGVHD (one 
limited, 11 extensive). At five years, the cumulative probabilities of aGVHD and cGVHD were $63 \%$ and $37 \%$, respectively.

\section{NRM}

A total of eight patients were alive at the time of this analysis, seven in complete remission (CR). The most common cause of death was disease relapse/progression. Causes of death were disease relapse/progression ( $\mathrm{n}=$ $27)$, GVHD $(n=2)$, sinusoidal obstruction syndrome (SOS) $(\mathrm{n}=3)$, Epstein-Barr virus associated post-transplant lymphoproliferative disorder $(\mathrm{n}=1)$, and adenovirus infection $(n=1)$. Of six patients with CNS lesion, five died of disease relapse/progression $(n=3)$, GVHD $(\mathrm{n}=1)$ and $\operatorname{SOS}(\mathrm{n}=1)$, and one was alive at last follow-up although another HCT was planned due to BM relapse post-transplant. At five years, the cumulative probability of NRM was $38 \%$. Nine patients died before day 30, and 18 patients died within the first 100 days post-HCT.

\section{LFS and OS}

A total of 22 of 33 evaluable patients attained a CR after the allo-HCT. The median follow-up of survivors was 85 months (range, 24-126 months). The five-year KaplanMeier estimates of LFS and OS were $17 \%$ and $19 \%$, respectively.

\section{Univariable analysis}

We analyzed the impact of pre- and post-transplant characteristics on OS after allo-HCT. The factors included age at transplant, sex, primary vs. secondary leukemia, cytogenetics at diagnosis, number of BM blasts, donor type, myeloablative vs. reduced-intensity conditioning, and presence or absence of acute and chronic GVHD. Results of univariable analysis for OS are summarized in Table 2 . In the univariable analyses of the impact of pre-transplant variables on OS, poorrisk cytogenetics, number of BM blasts (>26\%), MDS overt AML and CB as stem cell source were significantly associated with worse prognosis $(\mathrm{p}=.03, \mathrm{p}=.01, \mathrm{p}=$ .02 and $\mathrm{p}<.001$, respectively). In addition, based on a landmark analysis at 6 months post-transplant, the fiveyear Kaplan-Meier estimates of OS in patients with and without prior history of cGVHD were $64 \%$ and $17 \%$ (p $=.022$ ) respectively (Figure 1 ).

\section{Bivariable analysis}

We performed the landmark analyses at 6 months posttransplant, which classified patients according to significant pre-transplant factors including poor-risk cytogenetics, number of BM blasts, or secondary leukemia and their prior history of cGVHD at 6 months post-transplant. Results of bivariable analysis for OS are shown in
Table 2 Univariable analysis of impact of pre-transplant variables on overall survival

\begin{tabular}{|c|c|c|}
\hline Variable & $\begin{array}{l}\text { Survival } \\
(\% \text { at } 5 \text { y) }\end{array}$ & $\begin{array}{l}\text { Log rank } \\
P \text { value }\end{array}$ \\
\hline \multicolumn{3}{|l|}{ Age at allo-HCT } \\
\hline$<40$ & 28 & 0.055 \\
\hline$\geq 40$ & 6 & \\
\hline \multicolumn{3}{|l|}{ Diagnosis } \\
\hline MDS overt AML & 0 & 0.015 \\
\hline Others & 25 & \\
\hline \multicolumn{3}{|l|}{ Cytogenetics } \\
\hline intermediate & 35 & 0.013 \\
\hline poor & 5 & \\
\hline \multicolumn{3}{|l|}{ Marrow blasts at allo-HCT } \\
\hline$\leq 26$ & 33 & 0.013 \\
\hline$>26$ & 5 & \\
\hline \multicolumn{3}{|l|}{ Donor source } \\
\hline Umbilical cord blood & 0 & $<0.001$ \\
\hline Others & 22 & \\
\hline \multicolumn{3}{|l|}{ Conditioning } \\
\hline Intensified & 22 & 0.087 \\
\hline Standard & 42 & \\
\hline Reduced-intensity & 0 & \\
\hline $\begin{array}{l}\text { Reduced-intensity + cytoreductive } \\
\text { chemotherapy }\end{array}$ & 7 & \\
\hline
\end{tabular}

allo-HCT: allogeneic hematopoietic cell transplantation.

Figure 2, Figure 3 and Figure 4. The groups of patients with intermediate cytogenetics, marrow blast $\leq 26 \%$ or primary leukemia, who developed cGVHD less than 6 months after transplant, showed significantly or borderline significantly higher survival rates than those in the other groups $(\mathrm{p}=.039, \mathrm{p}=.147$, and $\mathrm{p}=.060$, respectively). The five-year Kaplan-Meier estimates of OS in the patients with intermediate cytogenetics, marrow blast $\leq 26 \%$ or primary leukemia in addition to prior history of cGVHD were $75 \%, 83 \%$, and $64 \%$, respectively.

\section{Discussion}

Our data showed that allo-HCT resulted in long-term disease remission and an eventual cure of active leukemia in a subset of de novo AML or ALL patients with marrow blast $\leq 26 \%$ and without poor-risk cytogenetics, possibly by graft-versus-leukemia (GVL) effects mediated through cGVHD.

A retrospective study with a large cohort using data reported to the Center for International Blood and Marrow Transplant Research demonstrated that pre-transplant variables delineated subgroups with different long- 

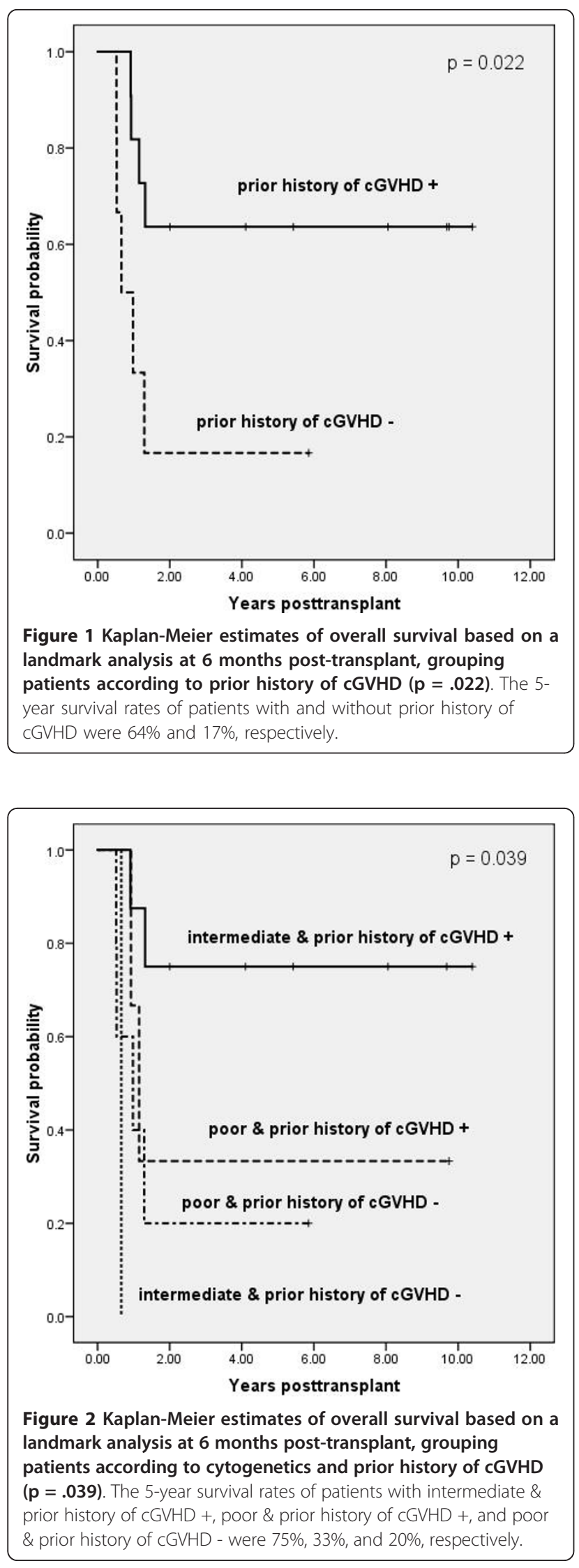

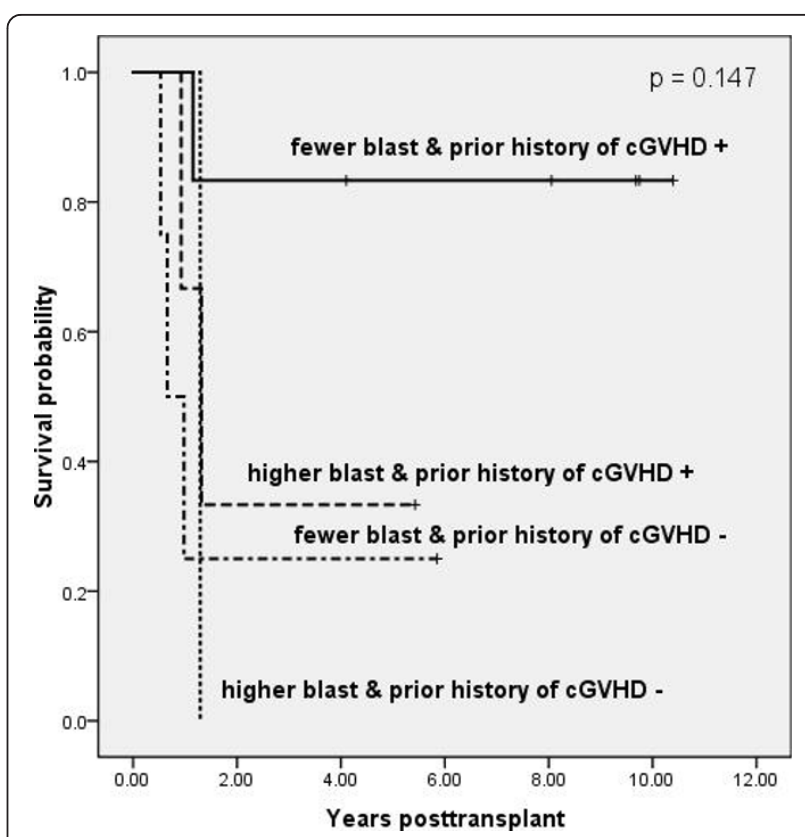

Figure 3 Kaplan-Meier estimates of overall survival based on a landmark analysis at 6 months post-transplant, grouping patients according to percent marrow blast ( $\leq$ or $>26 \%$ ) at baseline and prior history of cGVHD ( $p=.147)$. Patients with CNS lesion were not included in this analysis. The 5-year survival rates of patients with fewer blast \& prior history of cGVHD +, higher blast \& prior history of CGVHD +, and fewer blast \& prior history of cGVHD - were $83 \%, 33 \%$, and $25 \%$, respectively.

term allo-HCT outcomes in adult patients with acute leukemia not in remission [9]. However, they did not address the effect of cGVHD on survival. Baron et al. have reported that extensive cGVHD was associated with decreased risk of progression or relapse in patients with AML or MDS in complete remission at the time of nonmyeloablative HCT [16]. However, it remains unclear whether cGVHD is associated with long-term disease control in patients who have active leukemia at transplant. The results of the current study showed that GVL effects mediated by cGVHD may play a crucial role in long-term survival in or a cure of active leukemia, especially in patients without poor-risk cytogenetics. Further study on the possible relationship between cGVHD and GVL effects would be very helpful in the management of immunosuppressive treatment.

For patients who were ineligible for myeloablative conditioning due to comorbidities coupled with rapidly progressive leukemia, we administered sequential cytoreductive chemotherapy, followed by reduced-intensity conditioning for allo-HCT in order to reduce toxicity and obtain sufficient anti-leukemic efficacy. The utility of the combination of sequential cytoreductive chemotherapy and reduced-intensity conditioning for alloHCT was previously reported [17]. Our results did not 


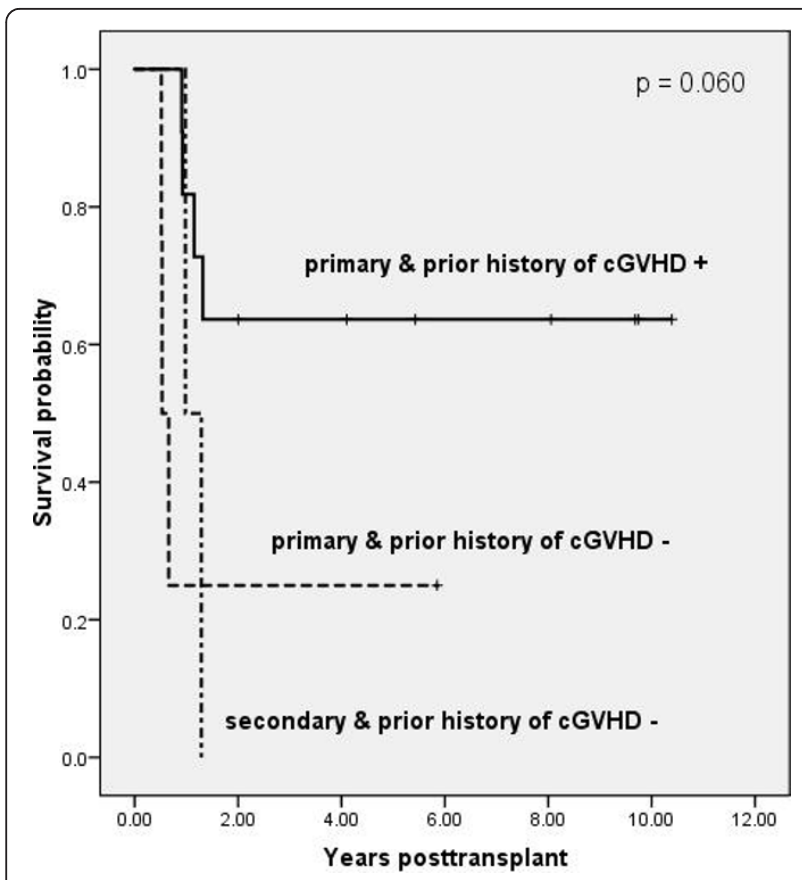

Figure 4 Kaplan-Meier estimates of overall survival based on a landmark analysis at 6 months post-transplant, grouping patients according to primary or secondary leukemia and prior history of cGVHD $(p=.060)$. The 5 -year survival rates of patients with primary \& prior history of CGVHD + and primary \& prior history of cGVHD - were $64 \%$ and $25 \%$, respectively.

show that this sequential regimen had an advantage in controlling active leukemia. However, we speculated that effective tumor reduction by individual chemotherapy and/or conditioning for allo-HCT to control disease until cGVHD subsequently occurred might also be important, particularly in rapidly proliferating leukemia. In contrast, intensive conditioning did not appear to be essential in relatively indolent leukemia, even with nonremission.

Based on our results, $\mathrm{CB}$ might be unsuitable as a source of stem cells for treatment of active leukemia at the time of allo-HCT. However, most patients receiving CBT could not wait for an unrelated donor search because their disease tended to be aggressive compared with those in the unrelated BM group. Thus, it is difficult to arrive at any conclusions about the best stem cell source for allo-HCT in patients in non-remission status based solely on our results.

Our study has several limitations. The results might be affected by an underlying selection bias due to the nature of retrospective data. Also, our study was limited by the small number of patients, the heterogeneity of the disease, the transplant procedure and the stem cell source. However, the major strengths of our study were that the follow-up period was sufficient with more than 5 years and the impact of cGVHD as well as pre-transplant factors on long-term survival were analyzed exclusively for subjects with active leukemia.

\section{Conclusion}

These data show that allo-HCT has the potential to cure active leukemia possibly via cGVHD, particularly in patients with favorable factors even when in non-remission. Further research is warranted to explore the essential factors contributing to the success of allo-HCT such as intensity of conditioning, and GVL effects mediated through cGVHD.

\section{Acknowledgements}

This work was supported by a Grant-in-Aid for Scientific Research from the Japanese Ministry of Education, Science, Sports, and Culture, and a grant from the Japanese Ministry of Health, Welfare, and Labour.

\section{Author details}

${ }^{1}$ Hematology, Graduate School of Medicine, Osaka City University, Osaka, Japan. ${ }^{2}$ Diagnostic Pathology, Graduate School of Medicine, Osaka City University, Osaka, Japan.

\section{Authors' contributions}

$\mathrm{HK}$ and HN designed the study and wrote the paper; HK analyzed results and created the figures; $\mathrm{MH}$ designed the research; $\mathrm{M}$ Nakamae and $\mathrm{YU}$ reviewed the patients' medical records and cleaned the data; $\mathrm{MO}$ reviewed the pathological specimens in this study; and $\mathrm{KH}, \mathrm{TN}, \mathrm{MM}, \mathrm{YH}, \mathrm{M}$ Nishimoto, $\mathrm{AH}, \mathrm{El}, \mathrm{Al}, \mathrm{MY}, \mathrm{MB}, \mathrm{HO}, \mathrm{RA}, \mathrm{MA}, \mathrm{YT}, \mathrm{KK}, \mathrm{TY}$ reviewed the results. All authors have read and approved the final manuscript.

\section{Competing interests}

The authors declare that they have no competing interests.

Received: 13 January 2011 Accepted: 10 April 2011

Published: 10 April 2011

\section{References}

1. Champlin R, Gale RP: Acute myelogenous leukemia: recent advances in therapy. Blood 1987, 69:1551-1562.

2. Biggs JC, Horowitz MM, Gale RP, Ash RC, Atkinson K, Helbig W, Jacobsen N, Phillips GL, Rimm AA, Ringdén $O$, et al: Bone marrow transplants may cure patients with acute leukemia never achieving remission with chemotherapy. Blood 1992, 80:1090-1093.

3. Sierra J, Storer B, Hansen JA, Bjerke JW, Martin PJ, Petersdorf EW, Appelbaum FR, Bryant E, Chauncey TR, Sale G, et al: Transplantation of marrow cells from unrelated donors for treatment of high-risk acute leukemia: the effect of leukemic burden, donor HLA-matching, and marrow cell dose. Blood 1997, 89:4226-4235.

4. Greinix HT, Reiter E, Keil F, Fischer G, Lechner K, Dieckmann K, Leitner G, Schulenburg A, Hoecker P, Haas OA, et al: Leukemia-free survival and mortality in patients with refractory or relapsed acute leukemia given marrow transplants from sibling and unrelated donors. Bone Marrow Transplant 1998, 21:673-678.

5. Wong R, Shahjahan M, Wang X, Thall PF, De Lima M, Khouri I, Gajewski J, Alamo J, Couriel D, Andersson BS, et al: Prognostic factors for outcomes of patients with refractory or relapsed acute myelogenous leukemia or myelodysplastic syndromes undergoing allogeneic progenitor cell transplantation. Biol Blood Marrow Transplant 2005, 11:108-114.

6. Oyekunle AA, Kröger N, Zabelina T, Ayuk F, Schieder H, Renges H, Fehse N, Waschke $\mathrm{O}$, Fehse $\mathrm{B}$, Kabisch $\mathrm{H}$, et al: Allogeneic stem-cell transplantation in patients with refractory acute leukemia: a long-term follow-up. Bone Marrow Transplant 2006, 37:45-50.

7. Brown RA, Wolff SN, Fay JW, Pineiro L, Collins RH Jr, Lynch JP, Stevens D, Greer J, Herzig RH, Herzig GP: High-dose etoposide, cyclophosphamide, and total body irradiation with allogeneic bone marrow transplantation for patients with acute myeloid leukemia in untreated first relapse: a 
study by the North American Marrow Transplant Group. Blood 1995, 85:1391-1395.

8. Brown RA, Wolff SN, Fay JW, Pineiro L, Collins RH Jr, Lynch JP, Stevens D, Greer J, Herzig RH, Herzig GP: High-dose etoposide, cyclophosphamide and total body irradiation with allogeneic bone marrow transplantation for resistant acute myeloid leukemia: a study by the North American Marrow Transplant Group. Leuk Lymphoma 1996, 22:271-277.

9. Duval M, Klein JP, He W, Cahn JY, Cairo M, Camitta BM, Kamble R, Copelan E, de Lima M, Gupta V, et al: Hematopoietic stem-cell transplantation for acute leukemia in relapse or primary induction failure. J Clin Oncol 2010, 28:3730-3738.

10. Slovak ML, Kopecky KJ, Cassileth PA, Harrington DH, Theil KS, Mohamed A, Paietta E, Willman CL, Head DR, Rowe JM, et al: Karyotypic analysis predicts outcome of preremission and postremission therapy in adult acute myeloid leukemia: a Southwest Oncology Group/Eastern Cooperative Oncology Group Study. Blood 2000, 96:4075-4083.

11. Moorman AV, Harrison CJ, Buck GA, Richards SM, Secker-Walker LM, Martineau M, Vance GH, Cherry AM, Higgins RR, Fielding AK, et al: Karyotype is an independent prognostic factor in adult acute lymphoblastic leukemia (ALL): analysis of cytogenetic data from patients treated on the Medical Research Council (MRC) UKALLXII/Eastern Cooperative Oncology Group (ECOG) 2993 trial. Blood 2007, 109:3189-3197.

12. Przepiorka D, Weisdorf $D$, Martin $P$, Klingemann HG, Beatty $P$, Hows J, Thomas ED: 1994 Consensus Conference on Acute GVHD Grading. Bone Marrow Transplant 1995, 15:825-828.

13. Vogelsang GB: How I treat chronic graft-versus-host disease. Blood 2001, 97:1196-1201.

14. Gooley TA, Leisenring W, Crowley J, Storer BE: Estimation of failure probabilities in the presence of competing risks: new representations of old estimators. Stat Med 1999, 18:695-706.

15. Storer BE: Statistical considerations in studies of late effects in HCT. Biol Blood Marrow Transplant 2009, 15(Suppl 1):25-28.

16. Baron F, Maris MB, Sandmaier BM, Storer BE, Sorror M, Diaconescu R, Woolfrey AE, Chauncey TR, Flowers ME, Mielcarek M, et al: Graft-versustumor effects after allogeneic hematopoietic cell transplantation with nonmyeloablative conditioning. I Clin Oncol 2005, 23:1993-2003.

17. Schmid C, Schleuning M, Schwerdtfeger R, Hertenstein B, MischakWeissinger E, Bunjes D, Harsdorf SV, Scheid C, Holtick U, Greinix H: Longterm survival in refractory acute myeloid leukemia after sequential treatment with chemotherapy and reduced-intensity conditioning for allogeneic stem cell transplantation. Blood 2006, 108:1092-1099.

doi:10.1186/1756-9966-30-36

Cite this article as: Koh et al:: Factors that contribute to long-term survival in patients with leukemia not in remission at allogeneic hematopoietic cell transplantation. Journal of Experimental \& Clinical Cancer Research 2011 30:36.

\section{Submit your next manuscript to BioMed Central and take full advantage of:}

- Convenient online submission

- Thorough peer review

- No space constraints or color figure charges

- Immediate publication on acceptance

- Inclusion in PubMed, CAS, Scopus and Google Scholar

- Research which is freely available for redistribution

Submit your manuscript at www.biomedcentral.com/submit
C Biomed Central 